\title{
On the Hodge Cycles of Prym Varieties
}

\author{
INDRANIL BISWAS
}

\begin{abstract}
We show that the Néron-Severi group of the Prym variety for a degree three unramified Galois covering of a hyperelliptic Riemann surface has a distinguished subgroup of rank three. For the general hyperelliptic curve, the algebra of Hodge cycles on the Prym variety is generated by this group of rank three.
\end{abstract}

Mathematics Subject Classification (2000): 14H40 (primary); 14C30 (secondary).

\section{1. - Introduction}

Let $X$ be a hyperelliptic Riemann surface. Let $f: Y \longrightarrow X$ be a unramified Galois covering with Galois group $\mathbb{Z} / 3 \mathbb{Z}$ with $Y$ connected. The Néron-Severi group of the Prym variety $\operatorname{Prym}(f)$ has a certain distinguished subgroup of rank three. This subgroup is constructed as follows. We first prove that $\operatorname{Prym}(f)$ is identified with $\operatorname{Pic}^{0}(Z) \times \operatorname{Pic}^{0}(Z)$, where $Z$ is a Riemann surface constructed from the covering datum (Lemma 3.1). The two projections and the addition law of $\operatorname{Pic}^{0}(Z)$ together give three maps from $\operatorname{Pic}^{0}(Z) \times \operatorname{Pic}^{0}(Z)$ to $\operatorname{Pic}^{0}(Z)$. The pull backs, by these three maps, to $\operatorname{Pic}^{0}(Z) \times \operatorname{Pic}^{0}(Z)=\operatorname{Prym}(f)$ of the natural polarization on $\operatorname{Pic}^{0}(Z)$ give the distinguished subgroup of the Néron-Severi group of $\operatorname{Prym}(f)$.

For the general hyperelliptic curve $X$, the algebra of Hodge cycles on $\operatorname{Prym}(f)$ is generated by this subgroup of rank three of $\mathrm{NS}(\operatorname{Prym}(f)$ ) (Theorem 4.1). In particular, for general hyperelliptic $X$, the Néron-Severi group $\mathrm{NS}(\operatorname{Prym}(f))$ is of rank three, and all the Hodge cycles on $\operatorname{Prym}(f)$ are algebraic.

We also consider degree three ramified coverings of an arbitrary compact Riemann surface. Under an assumption on the covering, the algebra of Hodge cycles of the Prym variety for the general Riemann surface is again generated by the Néron-Severi group (Lemma 5.1). 


\section{2. - Degree three covering of hyperelliptic curve}

Let $X$ be a connected smooth projective curve defined over the field of complex numbers. Assume that $g \geq 2$, where $g$ denotes the genus of $X$. Let

$$
f: Y \longrightarrow X
$$

be an étale covering map of degree $d$ with $Y$ being connected. We have the norm homomorphism

$$
N: \operatorname{Pic}^{0}(Y) \longrightarrow \operatorname{Pic}^{0}(X)
$$

of abelian varieties. We recall that for any line bundle $L$ on $Y$, the direct image $f_{*} L$ has a reduction of structure group to the monomial group (the group of $d \times d$ invertible complex matrices with exactly one nonzero entry in each row). For any $L \in \operatorname{Pic}^{0}(Y)$ the line bundle

$$
N(L) \in \operatorname{Pic}^{0}(X)
$$

is the one associated to $f_{*} L$ for the character of the monomial group defined by the permanent (the permanent of a matrix in the monomial group is the product of all its nonzero entries).

The connected component, containing the identity element, of the kernel of the homomorphism $N$ in (2.1) is called the Prym variety for $f$. The Prym variety for the map $f$ will be denoted by $\operatorname{Prym}(f)$.

Assume that the covering $f$ is Galois and that the Galois group for $f$ is abelian. Also, assume $X$ to be hyperelliptic. We have the following simple proposition.

PROPOSITION 2.1. The hyperelliptic involution of $X$ lifts to an involution of $Y$.

Proof. Let $\sigma \in \operatorname{Aut}(X)$ be the hyperelliptic involution. Let $\tilde{\sigma} \in$ Aut $\left(H_{1}(X, \mathbb{Z})\right)$ be the involution defined by the action of $\sigma$. It is straight-forward to check that $\tilde{\sigma}$ is the inversion homomorphism $\theta \longmapsto-\theta$. Consequently, $\tilde{\sigma}$ preserves the kernel of any homomorphism from $H_{1}(X, \mathbb{Z})$. In particular, it preserves the kernel of the natural homomorphism $H_{1}(X, \mathbb{Z}) \longrightarrow \mathrm{Gal}(Y / X)$, where $\operatorname{Gal}(Y / X)$ is the Galois group for the covering $f$. Note that since the Galois group is abelian, the projection $\pi_{1}(X) \longrightarrow \operatorname{Gal}(Y / X)$ induces a projection from $H_{1}(X, \mathbb{Z})$ to $\operatorname{Gal}(Y / X)$.

Now from the homotopy lifting property it follows immediately that $\sigma$ lifts to an automorphism of $Y$.

We can choose the lift of $\sigma$ to be an involution of $Y$. Indeed, take a Weierstrass point $x_{0} \in X$ (that is, fixed point for $\sigma$ ), and take any point $y_{0} \in f^{-1}\left(x_{0}\right)$. Let $\sigma_{Y}$ be the (unique) lift of $\sigma$ with $\sigma_{Y}\left(y_{0}\right)=y_{0}$. Since $\sigma_{Y} \circ \sigma_{Y} \in \operatorname{Gal}(Y / X)$ and $\sigma_{Y} \circ \sigma_{Y}\left(y_{0}\right)=y_{0}$, this lift $\sigma_{Y}$ must be an involution. This completes the proof of the proposition. 
After fixing a point $y_{0} \in f^{-1}\left(x_{0}\right)$ as in the proof of Proposition 2.1, the fiber $f^{-1}\left(x_{0}\right)$ gets identified with $\operatorname{Gal}(Y / X)$. Since the action of $\sigma$ on $H_{1}(X, \mathbb{Z})$ is multiplication by -1 , the action of the lift $\sigma_{Y}$ (the involution constructed in the proof of Proposition 2.1 that fixes $\left.y_{0}\right)$ on $f^{-1}\left(x_{0}\right)$ is the inversion homomorphism $\theta \longmapsto-\theta$ in terms of the identification of $f^{-1}\left(x_{0}\right)$ with $\operatorname{Gal}(Y / X)$.

Take any lift $\sigma^{\prime} \in \operatorname{Aut}(Y)$ of $\sigma$. So we have $\sigma^{\prime}=\gamma \circ \sigma_{Y}$, where $\gamma$ is some element of $\operatorname{Gal}(Y / X)$.

Now assume that $\operatorname{Gal}(Y / X) \cong \mathbb{Z} / 3 \mathbb{Z}$. From the above description of the action of $\sigma_{Y}$ on $f^{-1}\left(x_{0}\right)$ it can be deduced that the automorphism $\sigma^{\prime}=\gamma \circ \sigma_{Y}$ on $f^{-1}\left(x_{0}\right)$ has exactly one fixed point, namely $\gamma^{-1}\left(y_{0}\right)$. Indeed, it is easy to see that

$$
\delta \circ \sigma_{Y}=\sigma_{Y} \circ \delta^{-1}
$$

on $f^{-1}\left(x_{0}\right)$ for all $\delta \in \operatorname{Gal}(Y / X)$. As $\gamma^{2}=\gamma^{-1}$ and $\sigma_{Y}\left(y_{0}\right)=y_{0}$, from (2.2) it follows immediately that $\gamma^{-1}\left(y_{0}\right)$ is the unique fixed point for the action of $\gamma \circ \sigma_{Y}$ on $f^{-1}\left(x_{0}\right)$.

Since $\sigma_{Y}^{-1} \circ \gamma \circ \sigma_{Y} \in \operatorname{Gal}(Y / X)$, from the equality (2.2) on $f^{-1}\left(x_{0}\right)$ it follows that

$$
\gamma \circ \sigma_{Y}=\sigma_{Y} \circ \gamma^{-1}
$$

on $Y$. Note that (2.3) implies that $\gamma \circ \sigma_{Y}$ is an involution. Since $x_{0}$ is an arbitrary Weierstrass point of $X$, we have following proposition.

Proposition 2.2. Assume that $\operatorname{Gal}(Y / X) \cong \mathbb{Z} / 3 \mathbb{Z}$. Let $x_{0} \in X$ be a fixed point of the hyperelliptic involution $\sigma$ of $X$. Then for any lift $\sigma^{\prime} \in \operatorname{Aut}(Y)$ of $\sigma$, the action of $\sigma^{\prime}$ on $f^{-1}\left(x_{0}\right)$ has exactly one fixed point. Furthermore, $\sigma^{\prime}$ itself is an involution.

The genus of $Y$ is $3 g-2$, where $g$ is the genus of $X$. The Proposition 2.2 implies that there are exactly $2 g+2$ fixed points of the involution $\sigma^{\prime}$ (the number of Weierstrass points of $X$ ). This in turn implies that the quotient Riemann surface $Y / \sigma^{\prime}$ is of genus $g-1$.

\section{3. - Prym for degree three covering}

As before, let $f$ be an étale Galois covering of the hyperelliptic Riemann surface $X$ with Galois group $\operatorname{Gal}(Y / X) \cong \mathbb{Z} / 3 \mathbb{Z}$. Fix a lift $\sigma_{Y}$ of the hyperelliptic involution $\sigma$. Let

$$
Z:=Y / \sigma_{Y}
$$

be the quotient of $Y$ by the involution $\sigma_{Y}$ (see Proposition 2.2). 
Lemma 3.1. The Prym variety $\operatorname{Prym}(f)$ is canonically isomorphic to the self product $\operatorname{Pic}^{0}(Z) \times \operatorname{Pic}^{0}(Z)$, where $Z$ is the curve defined in (3.1).

Proof. Let $\gamma \in \operatorname{Gal}(Y / X)$ be a nontrivial element. So $\sigma^{\prime}:=\gamma \circ \sigma_{Y}$ is another lift of $\sigma$ distinct from $\sigma_{Y}$. It was noted in Proposition 2.2 that $\sigma^{\prime}$ is also an involution of $Y$. Let

$$
q_{1}: Y \longrightarrow Z_{1}:=Y / \sigma^{\prime}
$$

be the quotient map. We will show that $Z_{1}$ is isomorphic to $Z$ defined in (3.1).

Let

$$
q: Y \longrightarrow Z
$$

be the quotient map in (3.1). Take any point $z \in Z$. Let $\left\{y_{1}, y_{2}\right\}=q^{-1}(z) \subset Y$ (if $q$ is ramified over $z$, then $\left.y_{1}=y_{2}\right)$. In other words, $y_{2}=\sigma_{Y}\left(y_{1}\right)$.

Set $\sigma^{\prime \prime}:=\gamma \circ \gamma \circ \sigma_{Y}$, which is in fact the third lift of $\sigma$ (apart from $\sigma_{Y}$ and $\left.\sigma^{\prime}\right)$. Set $y_{1}^{\prime}:=\sigma^{\prime \prime}\left(y_{1}\right)$ and $y_{2}^{\prime}:=\sigma^{\prime \prime}\left(y_{2}\right)$. We will prove that

$$
\sigma^{\prime}\left(y_{1}^{\prime}\right)=y_{2}^{\prime} \text {. }
$$

To prove this, first note that

$$
\sigma^{\prime}\left(y_{1}^{\prime}\right)=\gamma \circ \sigma_{Y} \circ \sigma^{\prime \prime}\left(y_{1}\right)=\sigma_{Y} \circ \gamma^{-1} \circ \gamma \circ \gamma \circ \sigma_{Y}\left(y_{1}\right)=\sigma_{Y} \circ \gamma \circ \sigma_{Y}\left(y_{1}\right)
$$

because $\gamma \circ \sigma_{Y}=\sigma_{Y} \circ \gamma^{-1}$ (see (2.3)) and both $\sigma_{Y}$ and $\sigma^{\prime}$ are involutions (see Proposition 2.2). Now,

$$
\sigma_{Y} \circ \gamma \circ \sigma_{Y}\left(y_{1}\right)=\gamma^{-1} \circ \sigma_{Y} \circ \sigma_{Y}\left(y_{1}\right)=\gamma^{-1}\left(y_{1}\right)
$$

as $\sigma_{Y}$ is an involution. On the other hand,

$$
y_{2}^{\prime}=\sigma^{\prime \prime}\left(y_{2}\right)=\gamma \circ \gamma \circ \sigma_{Y} \circ \sigma_{Y}\left(y_{1}\right)=\gamma \circ \gamma\left(y_{1}\right)=\gamma^{-1}\left(y_{1}\right)
$$

because $\gamma^{3}=1$. Comparing (3.5), (3.6) and (3.7) we conclude that (3.4) is valid $\left(\right.$ as $\left.\sigma^{\prime}\left(y_{1}^{\prime}\right)=\sigma_{Y} \circ \gamma \circ \sigma_{Y}\left(y_{1}\right)=\gamma^{-1}\left(y_{1}\right)=y_{2}^{\prime}\right)$.

Note that since $\sigma^{\prime}$ is an involution, (3.4) implies that $\sigma^{\prime}\left(y_{2}^{\prime}\right)=y_{1}^{\prime}$. Consequently, there is a (unique) point $z_{1} \in Z_{1}$ such that

$$
q_{1}^{-1}\left(z_{1}\right)=\left\{y_{1}^{\prime}, y_{2}^{\prime}\right\} \subset Y
$$

where $q_{1}$ is defined in (3.2).

Let $T: Z \longrightarrow Z_{1}$ be the morphism that sends any point $z$ to the point $z_{1}$ constructed above from $z$. This map $T$ is clearly an isomorphism. Consequently, the curve $Z$ is isomorphic to $Z_{1}$.

Let

$$
F: \operatorname{Pic}^{0}(Z) \times \operatorname{Pic}^{0}\left(Z_{1}\right) \longrightarrow \operatorname{Pic}(Y)
$$


be the morphism defined by $\left(L, L_{1}\right) \longmapsto q^{*} L \otimes q_{1}^{*} L_{1}$, where $q$ and $q_{1}$ are defined in (3.3) and (3.2) respectively.

Since $Z$ is canonically isomorphic to $Z_{1}$ (the isomorphism $T$ is constructed above), to prove the lemma it suffices to show that the map $F$ defined in (3.8) is an isomorphism of $\operatorname{Pic}^{0}(Z) \times \operatorname{Pic}^{0}\left(Z_{1}\right)$ with the Prym variety $\operatorname{Prym}(f)$.

For that first note that we have a commutative diagram of maps

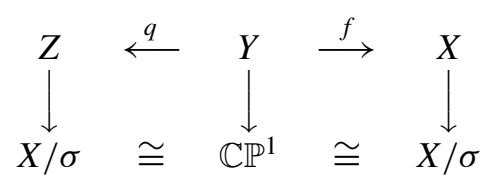

Take any line bundle $L \in \operatorname{Pic}^{0}(Z)$. Using the above diagram it follows immediately that the line bundle

$$
\bigotimes_{\gamma \in \operatorname{Gal}(Y / X)} \gamma^{*} q^{*} L
$$

on $Y$ descends to $X / \sigma$. Since $\operatorname{Pic}^{0}(X / \sigma)=0$, we conclude that $q^{*} L \in \operatorname{Prym}(f)$. (As $\operatorname{Pic}^{0}(Z)$ is connected and $q^{*} \mathcal{O}_{Z}=\mathcal{O}_{Y}$, it follows that $q^{*} L$ belongs to the component of the kernel of the norm map (defined in (2.1)) containing the identity element.)

Similarly, we have $q_{1}^{*} L_{1} \in \operatorname{Prym}(f)$ for any $L_{1} \in \operatorname{Pic}^{0}\left(Z_{1}\right)$. Consequently, the image of $F$ defined in (3.8) is contained in the subabelian variety $\operatorname{Prym}(f) \subset$ $\operatorname{Pic}(Y)$.

Since both $\operatorname{Prym}(f)$ and $\operatorname{Pic}^{0}(Z) \times \operatorname{Pic}^{0}\left(Z_{1}\right)$ are of dimension $2(g-1)$ with $g$ being the genus of $X$, to prove that $F$ is an isomorphism it is enough to show that the map $F$ is injective.

To prove that $F$ is injective we need to show that if

$$
\xi \in \operatorname{Prym}(f)
$$

is contained in the images of both $\operatorname{Pic}^{0}(Z)$ and $\operatorname{Pic}^{0}\left(Z_{1}\right)$, that is, there is $L \in \operatorname{Pic}^{0}(Z)$ and $L_{1} \in \operatorname{Pic}^{0}\left(Z_{1}\right)$ with $F(L)=F\left(L_{1}\right)=\xi$, then $L=0$ and $L_{1}=0$.

Since $\xi=F(L)$ (respectively, $\xi=F\left(L_{1}\right)$ ), the action of $\sigma_{Y}$ (respectively, $\left.\sigma^{\prime}\right)$ on $\operatorname{Pic}(Y)$ fixes $\xi$. Therefore, from the identity $\sigma^{\prime}=\gamma \circ \sigma_{Y}$ it follows that the action of $\gamma$ on $\operatorname{Pic}(Y)$ fixes $\xi$.

Now, $\gamma$, being nontrivial, is a generator of the Galois group $\operatorname{Gal}(Y / X)$. Therefore, both $L$ and $L_{1}$ descends to $X / \sigma$. Consequently, we have $L=0$ and $L_{1}=0$. This completes the proof of the lemma.

The isomorphism in Lemma 3.1 is canonical in the sense that no choices are involved in the construction of the isomorphism $F$. Note that if genus $(X)=1$, then clearly $\operatorname{Prym}(f)=e$.

The Hodge cycles on the general Prym variety will be described using the above isomorphism $F$. 


\section{4. - Hodge cycles on the general Prym}

From Lemma 3.1 it follows that the rank of the Néron-Severi group

$$
\mathrm{NS}(\operatorname{Prym}(f)):=H^{1,1}(\operatorname{Prym}(f)) \cap H^{2}(\operatorname{Prym}(f), 2 \pi \sqrt{-1} \mathbb{Z})
$$

is at least three. To explain this, let $\Theta$ denote the theta line bundle on $\operatorname{Pic}^{0}(Z)$. On $\operatorname{Pic}^{0}(Z) \times \operatorname{Pic}^{0}(Z)$ we have three line bundles $p_{1}^{*} \Theta, p_{2}^{*} \Theta$ and $s^{*} \Theta$, where

$$
p_{i}: \operatorname{Pic}^{0}(Z) \times \operatorname{Pic}^{0}(Z) \longrightarrow \operatorname{Pic}^{0}(Z)
$$

is the projection to the $i$-th factor and $s$ is the group law on the abelian group $\operatorname{Pic}^{0}(Z)$. It is easy to see that the rank of the subgroup of the Néron-Severi group of $\operatorname{Pic}^{0}(Z) \times \operatorname{Pic}^{0}(Z)$ generated by the first Chern class of these three line bundles is three. Now, using the isomorphism in Lemma 3.1, we have a rank three subgroup of NS(Prym $(f))$.

Let

$$
\mathcal{C} \subset \bigoplus_{p \geq 0} H^{p, p}(\operatorname{Prym}(f)) \cap H^{2 p}\left(\operatorname{Prym}(f),(2 \pi \sqrt{-1})^{p} \mathbb{Q}\right)
$$

denote the subalgebra of the algebra of Hodge cycles on $\operatorname{Prym}(f)$ generated by the above three elements of the Néron-Severi group and $1 \in H^{0}(\operatorname{Prym}(f), \mathbb{Q})$.

THEOREM 4.1. For the general hyperelliptic Riemann surface $X$, the subalgebra $\mathcal{C}$ defined in (4.1) coincides with the space of all Hodge cycles on $\operatorname{Prym}(f)$.

Proof. Consider the family of abelian varieties $\operatorname{Prym}(f)$ as $f$ runs over all connected étale Galois covers of degree three of all hyperelliptic curves of genus $g$. Using Lemma 3.1 it can be deduced that the monodromy of this family is Zariski dense in $\operatorname{Sp}(2(g-1), \mathbb{C})$ for the diagonal action of $\operatorname{Sp}(2(g-$ $1), \mathbb{C})$ on $\mathbb{C}^{2(g-1)} \oplus \mathbb{C}^{2(g-1)}$. Indeed, using the decomposition of the Prym variety $\operatorname{Prym}(f)$ given in Lemma 3.1 it follows immediately that the monodromy is contained in $\operatorname{Sp}(2(g-1), \mathbb{C})$ (with $\operatorname{Sp}(2(g-1), \mathbb{C})$ acting diagonally on $\left.\mathbb{C}^{2(g-1)} \oplus \mathbb{C}^{2(g-1)}\right)$. Now using degeneration of a hyperelliptic curve it can be seen that the Zariski closure coincides with $\operatorname{Sp}(2(g-1), \mathbb{C})$.

To prove the above assertion that the Zariski closure is indeed $\operatorname{Sp}(2(g-$ $1), \mathbb{C})$, let $E$ be an elliptic curve and $\tau$ an element of $E$ of order three. Let $X_{0}$ be an arbitrary smooth hyperelliptic curve of genus $g-1$. Fix a Weierstrass point $x_{0}$ of $X_{0}$. Set

$$
Y=E \cup X_{0} \cup X_{0} \cup X_{0}
$$

to be the tree-like nodal curve obtained by identifying the point $x_{0}$ in the $i$-th component $X_{0}, i \in[1,3]$, with the point $i \tau$ of $E$. So $\mathbb{Z} / 3 \mathbb{Z}$ acts on $Y$ using translation by $\tau$ on $E$ and using cyclic permutation of the three ordered copies of $X_{0}$. The quotient, curve, which we will denote by $X$, has two irreducible components, namely $E /\langle\tau\rangle$ and $X_{0}$, and both components are smooth. The 
quotient $X$ has exactly one singular point (the identification of $0 \in E /\langle\tau\rangle$ with $\left.x_{0}\right)$. Clearly, the Prym variety for this covering $Y \longrightarrow X$ is $\operatorname{Pic}^{0}\left(X_{0}\right) \times \operatorname{Pic}^{0}\left(X_{0}\right)$. It is well-known that the monodromy for hyperelliptic curves of genus $g-1$ is Zariski dense in $\operatorname{Sp}(2(g-1), \mathbb{C})$ (c.f. [BP]). This immediately implies the above assertion that the monodromy for the family of Prym varieties is Zariski dense in $\operatorname{Sp}(2(g-1), \mathbb{C})$ for the diagonal action of $\operatorname{Sp}(2(g-1), \mathbb{C})$ on $\mathbb{C}^{2(g-1)} \bigoplus \mathbb{C}^{2(g-1)}$.

Consider the action of $\operatorname{Sp}(2(g-1), \mathbb{C})$ on the exterior algebra $\Lambda\left(\mathbb{C}^{2(g-1)}\right.$ $\left.\oplus \mathbb{C}^{2(g-1)}\right)$ induced by the diagonal action on $\mathbb{C}^{2(g-1)} \oplus \mathbb{C}^{2(g-1)}$. The algebra of invariants for this action is generated by the three elements in $\bigwedge^{2}\left(\mathbb{C}^{2(g-1)}\right.$ $\left.\bigoplus \mathbb{C}^{2(g-1)}\right)$ constructed using the standard symplectic structure on $\mathbb{C}^{2(g-1)}[\mathrm{Ho}$, p. 543, Theorem 2]. These three elements in $\bigwedge^{2}\left(\mathbb{C}^{2(g-1)} \oplus \mathbb{C}^{2(g-1)}\right)$ correspond to the three elements in the Néron-Severi group of $\operatorname{Pic}^{0}(Z) \times \operatorname{Pic}^{0}(Z)$ described earlier.

Given any irreducible $\operatorname{Sp}(2(g-1), \mathbb{C})$-submodule $W$ of $\bigwedge\left(\mathbb{C}^{2(g-1)} \oplus \mathbb{C}^{2(g-1)}\right)$, either $W$ is contained in

$$
\left(\wedge\left(\mathbb{C}^{2(g-1)} \oplus \mathbb{C}^{2(g-1)}\right)\right)^{\mathrm{Sp}(2(g-1), \mathbb{C})}
$$

(the algebra of invariants for the action of the symplectic group) or $W$ contains some vectors that are not of $(p, p)$-type for the Hodge type decomposition [BN, Theorem 3.2]. This implies that for the general Prym variety, the space all Hodge cycles coincide with $\mathcal{C}$ (see [BN, Theorem 5.1]). This completes the proof of the theorem.

In the next section we will consider a class of ramified covers of degree there that are not Galois.

\section{5. - Degree three ramified coverings}

Let $X$ be a connected smooth projective curve defined over $\mathbb{C}$ of genus $g$ and

$$
f_{0}: Z \longrightarrow X
$$

a covering of degree two, possibly ramified, with $Z$ connected and smooth. Take two distinct points $x_{1}, x_{2} \in X$ and a smooth embedding

$$
\gamma:[0,1] \longrightarrow X
$$

with $\gamma(0)=x_{1}$ and $\gamma(1)=x_{2}$. Assume that the image of $\gamma$ does not intersect the divisor on $X$ over which the covering $f_{0}$ in (5.1) is ramified. In particular, neither of $x_{1}$ and $x_{2}$ is a ramification point for $f_{0}$. Consider

$$
f_{0}^{-1}(\gamma([0,1])) \subset Z
$$


which is a disjoint union of two copies of $\gamma([0,1])$. Choose one of the two arcs. So $\gamma$ defines an embedding of $[0,1]$ in $Z$ with this chosen arc as the image. This embedding of $[0,1]$ in $Z$ will be denoted by $\gamma_{Z}$.

Using the above data, we have a degree three covering of $X$. To explain this, set

$$
Y=\left(Z \backslash \gamma_{Z}([0,1])\right) \cup(X \backslash \gamma((0,1))) \cup(0,1) \cup(0,1)
$$

and attach one side of $\gamma_{Z}([0,1])$ in $Z$ and the opposite side of $\gamma([0,1])$ in $X$ along a copy of $(0,1)$. This gives a ramified covering map

$$
f: Y \longrightarrow X
$$

of degree three which is defined using $f_{0}$ (defined in (5.1)) over $Z \backslash \gamma_{Z}([0,1])$ and using the inclusion map of $X \backslash \gamma((0,1))$ in $X$ over $X \backslash \gamma((0,1)) \subset Y$. Therefore, $f$ is ramified over $x_{1}$ and $x_{2}$ of index 1 apart from having the ramifications coming from $f_{0}$.

For the dependence on $\gamma$ of the pair $Y$ and $f$, it is easy to see that if $\gamma_{1}$ is another arc connecting $x_{1}$ with $x_{2}$ which is homotopic to $\gamma$ (fixing $x_{1}$ and $x_{2}$ ), then the corresponding Riemann surface and the covering map are canonically identified with $Y$ and $f$ respectively. In other words, the above construction depends only on the homotopy class of $\gamma$ (with fixed end points).

The covering map $f$ in (5.2) does not admit any nontrivial deck transformation. Indeed, this is an immediate consequence of the fact that over the point $x_{1} \in X$ there is a unramified point and a ramified point of index 1 .

It is easy to see that the moduli space of all coverings (without fixing $X$ ) of degree three of the above type is irreducible. Fixing $X, f_{0}$ and $x_{1}$, if the point $x_{2}$ approaches $x_{1}$ and $\gamma$ approaches the constant map, then in the limit, the base of the covering map becomes the nodal curve $X \cup \mathbb{C P}^{1}$ with $x_{1}$ as the unique singular point. Then the covering curve is the tree-like nodal curve

$$
X \cup Z \cup \mathbb{C P}^{1} \cup \mathbb{C P}^{1}
$$

with one $\mathbb{C P}^{1}$ touching $Z$ at the unramified point over $x_{1}$ (the other point in $Z$ over $x_{1}$ not in the image of $\gamma_{Z}$ ) and the other $\mathbb{C P}^{1}$ touching $X$ and $Z$ at $x_{1}$ and the ramified point over $x_{1}$ respectively; so there are exactly three singular points.

We now consider the family of all coverings of degree three of singular curves obtained this way. So the target of such a covering map $f$ is a connected nodal curve of the form

$$
X^{\prime}=X \cup \mathbb{C P}^{1}
$$

with one singular point, where $X$ is some smooth curve of genus $g$. The domain of $f$ is

$$
Y^{\prime}=Z \cup X \cup \mathbb{C P}^{1} \cup \mathbb{C P}^{1}
$$


where

$$
f_{0}=\left.f\right|_{Z}: Z \longrightarrow X
$$

is a (possibly ramified) double cover of $X$ as above; the curve $Y^{\prime}$ has exactly three singular points, and they are of the above type.

For this covering map $f$ between singular curves, since

$$
\operatorname{Prym}(f)=\operatorname{Pic}^{0}(X) \times \operatorname{Prym}\left(f_{0}\right),
$$

the Mumford-Tate group of $\operatorname{Prym}(f)$ is contained in $\operatorname{Sp}(2 g, \mathbb{C}) \times \operatorname{Sp}\left(2 g^{\prime}, \mathbb{C}\right)$, where $g^{\prime}=\operatorname{dim}_{\mathbb{C}} \operatorname{Prym}\left(f_{0}\right)$. Consider the moduli space of all such coverings.

LEMMA 5.1. For the general point of the moduli space of all coverings of the above type, the Mumford-Tate group of $\operatorname{Prym}(f)$ coincides with $\operatorname{Sp}(2 g, \mathbb{C}) \times$ $\operatorname{Sp}\left(2 g^{\prime}, \mathbb{C}\right)$.

Proof. Theorem 5 of [BP] (see [BP, p. 38]) says that the projection of the Mumford-Tate group of $\operatorname{Prym}(f)$ to the factor $\operatorname{Sp}\left(2 g^{\prime}, \mathbb{C}\right)$ is surjective for the general point of the moduli space. On the other hand, the projection of the Mumford-Tate group of $\operatorname{Prym}(f)$ to the factor $\operatorname{Sp}(2 g, \mathbb{C})$ is also surjective for the general point of the moduli space since the mapping class group for the general smooth genus $g$ curve surjects on to $\operatorname{Sp}(2 g, \mathbb{C})$.

Now we need the following simple group theoretic result for the proof of Lemma 5.1.

Proposition 5.2. Let $G$ be a connected Lie subgroup of $\operatorname{Sp}(2 m, \mathbb{C}) \times \operatorname{Sp}(2(m+$ $n), \mathbb{C})$, with $n>0$, such that the natural projections of $G$ to both $\operatorname{Sp}(2 m, \mathbb{C})$ and $\operatorname{Sp}(2(m+n), \mathbb{C})$ are surjective. Then $G$ coincides with $\operatorname{Sp}(2 m, \mathbb{C}) \times \operatorname{Sp}(2(m+n), \mathbb{C})$.

PROOF. To prove the proposition, consider the exact sequence

$$
e \longrightarrow \mathcal{K} \longrightarrow G \longrightarrow \mathrm{Sp}(2 m, \mathbb{C}) \longrightarrow e
$$

obtained from the projection $\operatorname{Sp}(2 m, \mathbb{C}) \times \operatorname{Sp}(2(m+n) \longrightarrow \operatorname{Sp}(2 m, \mathbb{C})$. Note that $\mathcal{K} \neq e$ as $n \geq 1$.

Identify $\operatorname{Sp}(2(m+n), \mathbb{C})$ with its image in $\operatorname{Sp}(2 m, \mathbb{C}) \times \operatorname{Sp}(2(m+n), \mathbb{C})$ for by the map that sends any $h$ to $(e, h)$. Using this identification, $\mathcal{K}$ in (5.6) is a subgroup of $\operatorname{Sp}(2(m+n), \mathbb{C})$.

We will now show that $\mathcal{K}$ is a normal subgroup of $\operatorname{Sp}(2(m+n), \mathbb{C})$.

For any $h \in \operatorname{Sp}(2(m+n), \mathbb{C})$, take $\bar{h} \in G$ that projects to $h$ (recall the hypothesis that the projection from $G$ is surjective). For any $k \in \mathcal{K}$, consider $\bar{h} k \bar{h}^{-1} \in \mathcal{K}$. It is straight-forward to check that $\bar{h} k \bar{h}^{-1}$ commutes with the factor $\operatorname{Sp}(2 m, \mathbb{C})$. Therefore, it follows that $h k h^{-1} \in \mathcal{K}$.

Since $\mathcal{K} \neq e$ is a normal subgroup of $\operatorname{Sp}(2(m+n), \mathbb{C})$ of positive dimension, we conclude that $\mathcal{K}=\operatorname{Sp}(2(m+n), \mathbb{C})$. In view of the given condition that $G$ surjects on to $\operatorname{Sp}(2 m, \mathbb{C})$, from this it follows immediately that $G=\operatorname{Sp}(2 m, \mathbb{C})$ $\times \operatorname{Sp}(2(m+n), \mathbb{C})$. This completes the proof of the proposition. 
Continuing with the proof of Lemma 5.1, first note that if the number of ramification points of the covering map $f_{0}=\left.f\right|_{Z}: Z \longrightarrow X$ in (5.4) is different from two, then $g \neq g^{\prime}$.

Since the Mumford-Tate group of $\operatorname{Prym}(f)$ projects surjectively to both $\operatorname{Sp}\left(2 g^{\prime}, \mathbb{C}\right)$ and $\operatorname{Sp}(2 g, \mathbb{C})$ for the general $f$, from Proposition 5.2 it follows immediately that if the number of ramification points of the covering map $f_{0}$ is different from two, then the Mumford-Tate group of $\operatorname{Prym}(f)$ coincides with $\operatorname{Sp}\left(2 g^{\prime}, \mathbb{C}\right) \times \operatorname{Sp}(2 g, \mathbb{C})$ for the general $f$.

For the remaining case where $f_{0}$ has exactly two ramification points, first note that from the proof of Proposition 5.2 it follows that a connected Lie subgroup $G$ of $\operatorname{Sp}(2 m, \mathbb{C}) \times \operatorname{Sp}(2 m, \mathbb{C})$ that projects surjectively to both the factors must coincide with $\operatorname{Sp}(2 m, \mathbb{C}) \times \operatorname{Sp}(2 m, \mathbb{C})$ unless it is the graph of some automorphism of $\operatorname{Sp}(2 m, \mathbb{C})$.

Fix $X^{\prime}$ as in (5.3). Let $y_{1}$ and $y_{2}$ be two points of $X$ over which $f_{0}$ in (5.4) is ramified. Now, fixing $X^{\prime}$ move the pair of ramification points $y_{1}$ and $y_{2}$ in a (real) one-parameter family so that they interchange positions. For example, take a smooth embedding $\psi$ of the disk

$$
D^{2}:=\left\{\left.z \in \mathbb{C}|| z\right|^{2} \leq 1\right\}
$$

in $X$ with $\psi(1)=y_{1}$ and $\psi(-1)=y_{2}$. Now for $\theta \in[0,1]$ set $y_{1}(\theta)=$ $\psi(\exp (\pi \sqrt{-1} \theta))$ and $y_{2}(\theta)=\psi(-\exp (\pi \sqrt{-1} \theta))$.

Consider the closed loop in the moduli space obtained by this family parametrized by $[0,1]$. For this family, the monodromy of $H^{*}(\operatorname{Prym}(f), \mathbb{C})$ is an element of $\operatorname{Sp}(2 g, \mathbb{C}) \times \operatorname{Sp}\left(2 g^{\prime}, \mathbb{C}\right)$ which projects to the identity element in $\operatorname{Sp}(2 g, \mathbb{C})$ (as $X$ is kept fixed) and project to a nontrivial element in $\operatorname{Sp}\left(2 g^{\prime}, \mathbb{C}\right)$ (as $H^{1}(Z, \mathbb{C})$ has nontrivial monodromy).

Therefore, the Mumford-Tate group for the general $f$ cannot be a graph of an automorphism of $\operatorname{Sp}(2 g, \mathbb{C})$. This completes the proof of the lemma.

Consider the covering map $f: Y \longrightarrow$ constructed in (5.2) using $f_{0}$ : $Z \longrightarrow X$ in (5.1). We have $\operatorname{Prym}(f)=\operatorname{Pic}^{0}(X) \times \operatorname{Prym}\left(f_{0}\right)$ (see (5.5)). On the other hand, for the covering $f_{0}, \operatorname{Pic}^{0}(Z)$ is isogenus to $\operatorname{Pic}^{0}(X) \times \operatorname{Prym}\left(f_{0}\right)$. Therefore, $\operatorname{Pic}^{0}(Z)$ is isogenus to $\operatorname{Prym}(f)$. Since the Mumford-Tate group of $\operatorname{Prym}(f)$ coincides with $\operatorname{Sp}(2 g, \mathbb{C}) \times \operatorname{Sp}\left(2 g^{\prime}, \mathbb{C}\right)$ for the general covering of the type $f_{0}$ (Lemma 5.1), we conclude that the Mumford-Tate group of $\operatorname{Pic}^{0}(Z)$ coincides with $\operatorname{Sp}(2 g, \mathbb{C}) \times \operatorname{Sp}\left(2 g^{\prime}, \mathbb{C}\right)$ for the general covering of the type $f_{0}$ (Mumford-Tate group). The Mumford-Tate group of two isogenus abelian varieties coincide. For a product of abelian varieties whose Mumford-Tate group is the full group (product of symplectic groups of corresponding dimensions), the algebra of all Hodge cycles on it is generated by the Hodge cycles on the individual factors. Consequently, we have the following corollary on the Jacobian of the general double cover (with arbitrary ramification).

COROLLARY 5.3. For the moduli space of double covers of connected smooth projective curves of genus $g$ (with fixed arbitrary ramification), the Néron-Severi group of the Jacobian of the general double cover is generated by two elements; the 
two elements are obtained from the decomposition (up to isogeny) of the Jacobian of a covering curve. Furthermore, the Néron-Severi group generates the algebra of Hodge cycles (of positive degree) on the Jacobian of the general double cover.

Now consider the family of degree three covering maps as in (5.2) between smooth curves considered at the beginning of this section. Degenerating such covers to covering maps between singular curves (by moving $x_{2}$ close to $x_{1}$ ), and then using Lemma 5.1 we have the following corollary.

COROLLARY 5.4. The Néron-Severi group of $\operatorname{Prym}(f)$ for the general covering $f$ (as in (5.2)) generates the algebra of all Hodge cycles (of positive degree) on $\operatorname{Prym}(f)$.

\title{
REFERENCES
}

[BN] I. Biswas - M. S. NARASIMHAN, Hodge classes of moduli spaces of parabolic bundles over the general curve, J. Algebraic Geom. 6 (1997), 697-715.

[BP] I. Biswas - K. H. Paranjape, The Hodge conjecture for general Prym varieties, Jour. Alg. Geom. 11 (2002), 33-39.

[Ho] R. E. Howe, Remarks on classical invariant theory, Trans. Amer. Math. Soc. 313 (1989), 539-570.

\author{
School of Mathematics \\ Tata Institute of Fundamental Research \\ Homi Bhabha Road \\ Mumbai 400005, India \\ indranil@math.tifr.res.in
}

